\title{
Line scale measurement using image registration
}

\author{
P.B. Costa ${ }^{1,2, \star}$, A. Marques ${ }^{3}$, F.O. Baldner ${ }^{1,2}$, and F.R. Leta ${ }^{2}$ \\ 1 National Institute of Metrology, Quality and Technology (INMETRO), Av. Nossa Senhora das Graças 50, CEP 25250-020, \\ Duque de Caxias-RJ, Brazil \\ 2 Mechanical Engineering Post-Graduation Program, Universidade Federal Fluminense (UFF), Rua Passo da Patria 156, \\ CEP 24210-240, Niterói-RJ, Brazil \\ 3 Mechanical Engineering Post-Graduation Program, Universidade Federal do Paraná (UFPR), Jardim das Américas, \\ CEP 81531-990, Curitiba-PR, Brazil
}

Received: 28 March 2013 / Accepted: 16 July 2013

\begin{abstract}
Currently, most advances in dimensional metrology might be seen by the evolution of noncontact measurement (optical measurements), in order to provide traceability for different areas that needs to calibrate microscopes or optical measure machines. In a similar way, the use of image processing techniques for the measuring of objects has been the subject of recent studies in computer vision and image metrology. In the attempt to meet the requirements and demands for high accuracy dimensional metrology with image processing techniques, this work will present the application of the image registration technique for the measurement of line scales. In the conventional calibration, the scales are measured in preestablished points, generally in intervals of $10 \%$ of the total scale length. With this application, it becomes possible to provide results for all the scale marks quickly and automatically, whereas in the conventional method it would require more time and, thus, a higher cost for the fulfillment of this measurement.
\end{abstract}

Keywords: Dimensional metrology; line scale; image processing; image registration

\section{Introduction}

Line scales are material standards mostly used for the verification and calibration of microscopes, and non-contact measurement systems. This type of measurement requires that line scales are calibrated with high accuracy level. In the last two decades National Institutes of Metrology have been developing research on line scale measurement in order to meet this requirement [1-4], However, these researches have the purpose of reducing the measurement uncertainty through improvements in displacement measuring systems or through error compensation of movement systems and not exactly by using image processing techniques. In such systems, these techniques are used only for identification of the markings throughout the scale, not to extract measurements.

The calibration of a line scale consists in determining the distance between the centers of two consecutive marks [5]. The distance between two marks of a line scale can vary from $1 \mathrm{~mm}$ up to $0.01 \mathrm{~mm}$. In the National Institute of Metrology, Quality and Technology from Brazil, Inmetro, this calibration is accomplished by dividing the scale in intervals of $10 \%$ of the total length of the scale. However, in some cases, other points can be measured for calibration.

\footnotetext{
* Correspondence: pbcosta@inmetro.gov.br
}

This type of standard may be calibrated every four years depending on the frequency in which it is used. During this period, if any characteristic in the system is changed or the user needs to use a scale in a different point other than the ones contained in the certificate, he will lose the traceability of the system. In a line scale the errors for each measured length are completely independent, i.e., it does not allow interpolation of the errors along the scale to find intermediate values which were not included in the calibration certificate.

In order to provide the user with traceability for every point, the line scale should be measured in its entirety. However, such a calibration is impracticable due to its high cost and the time spent in measurement, since users rarely use more than one or two points of a scale.

One way of meeting the necessity of obtaining results for all the lengths of the scale without increasing cost or making the process exceedingly long is by using the image registration technique. This technique is mainly used in the processing of biological images or in the construction of maps.

This work describes the implementation of a new methodology for calibrating line scales in which, by using the image registration technique, a calibration certificate may present results for the entire line scale length. By using image registration, the scale can be measured in all its points without the need of using a displacement 
measuring system with an interferometric laser. This new methodology is being developed with the purpose of providing a new calibration system in Inmetro, for the users of line scales in Brazil.

\section{Image registration}

The technique of image registration appears from the need of assembling a continuous image from several images acquired either at different times or sensors. This procedure is accomplished through redundant points in different images [6]. That is, points that may be viewed in two different images must now occupy the same space in a new image, relocating all the other points of the images. In this paper we used a process known as Image Mosaicking [7], which consists in joining, in one picture, several other smaller ones. This method is frequently used for generating maps [8] and panoramic pictures [9]. The steps for the process of image registration are, in general:

a. Pre-processing: In the first step the image is prepared in order to make the feature selection easier. This preparation might be in the form of noise reduction, image segmentation, edge detection or any other modification that may ease its further processing.

b. Feature selection: The selected features are the information that will be used from one image to identify its correspondence with the next image. These characteristics can be lines, curves, areas, edges, etc.

c. Feature correspondence: The correspondence between characteristics is accomplished by using the characteristics selected from the first image and searching for them in the second image. The method used is chosen accordingly to the type of characteristic used. However, on both images, points must be selected to match the characteristics, even if they are lines, curves or areas.

d. Determination of a transformation function: On this step, a function must be created to relate the coordinates of the corresponding points in both images. This function must be adapted in accordance with the number of points and the displacements originated from these points.

e. Resampling: After the determination of the coordinates by the transformation function, a new image is generated by fusing both analyzed images.

\section{Method}

The registration technique applied on a line scale calibration consists of acquiring images of the scale from the beginning until the end of the markings, keeping some redundant markings between the photos. Thus, it is possible to reconstruct the scale, without any sort of definition loss, in just one image.

From the recorded image of the entire scale, then it becomes possible to measure all its distances. However, this procedure causes an increase in measurement uncertainty

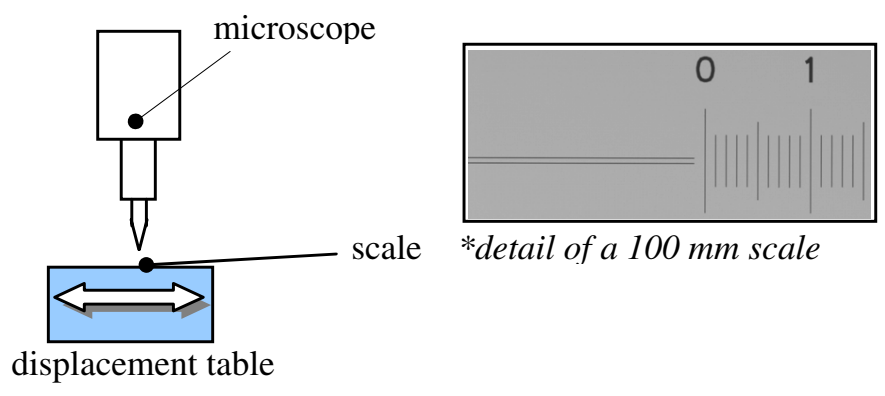

Fig. 1. Sketch of the equipment.

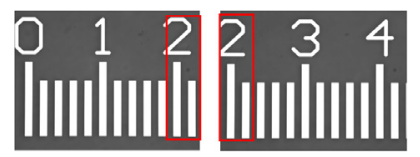

Fig. 2. Common region in two consecutive images.

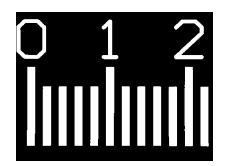

Fig. 3. Image segmentation result.

in comparison to the uncertainties obtained through the others conventional calibration methods.

The application of the registration technique in scale measurement follows some steps of a normal process of computer vision. The image acquisition was performed using a CCD Camera coupled to the measuring microscope with magnifications from $25 \times$ up to $1000 \times$. The camera used has a resolution of $768 \times 576$ with a Firewire interface. The scale is mounted over the displacement table to acquire the images as seen in Figure 1.

For the technique to display good results, in the image acquisition step, it is always important to have a common region on two consecutive images, as seen in Figure 2.

In the segmentation step, the goal is to find variations or discontinuities in the images so it will be possible to identify the scale marks. This identification is necessary since the position of the marks is used as a reference for the application of the registration. The image segmentation was performed based on its respective histogram, consisting on choosing a threshold and the pixels which have intensities lower than this threshold are turned to 0 (black) and the ones with intensities higher are turned to 255 (white) [10]. In order to choose an ideal value for this threshold, several values where tested and the one that presented the best results was the value of $50 \%$ of the image intensity. While there are several methods more advanced for image segmentation, an empiric solution could be applied to this work due to its histogram having well defined color sections. The segmentation result is shown in Figure 3.

In the registration process, the characteristics extraction is the key point for this methodology. In this stage, the algorithm must identify the redundant points in the sequenced images. 


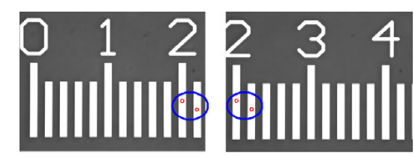

Fig. 4. Points used for the image registration.

The selected points are the geometric centers of the marks (shown in red), which are repeated on the pictures, as shown in Figure 4. The algorithm performs a scanning in the image searching for the center of the marks in both images and storing the $x$ and $y$ coordinates of the center of each mark.

Once the center coordinates values of each mark are visually perceived in both pictures, it is possible to perform the image registration. The registration is done following this procedure:

a. The coordinates of the two marks' centers of the first image are stored as: $x_{1}, y_{1}, x_{2} e y_{2}$.

b. On the second image, the coordinates of the same marks of the first picture are stored as: $X_{1}, Y_{, 1}, X_{2} e Y_{2}$.

c. A linear system is generated with the coordinates from the first and second image, as show in equation (1).

$$
\left\{\begin{array}{l}
X_{1}=a_{11}+a_{21} x_{1} \\
Y_{1}=a_{31}+a_{41} y_{1} \\
X_{2}=a_{11}+a_{21} x_{2} \\
Y_{2}=a_{31}+a_{41} y_{2}
\end{array}\right.
$$

The system is solved by its expansion in matrix form, as presented in equation (2).

$$
\left[\begin{array}{l}
X_{1} \\
Y_{1} \\
X_{2} \\
Y_{2}
\end{array}\right]=\left[\begin{array}{llll}
1 & 1 & 0 & 0 \\
0 & 0 & 1 & 1 \\
1 & 1 & 0 & 0 \\
0 & 0 & 1 & 1
\end{array}\right]\left[\begin{array}{l}
a_{11} \\
a_{21} \\
a_{31} \\
a_{41}
\end{array}\right]
$$

Renaming the matrices (Eq. (3)):

$$
A=\left[\begin{array}{l}
X_{1} \\
Y_{1} \\
X_{2} \\
Y_{2}
\end{array}\right] \quad B=\left[\begin{array}{llll}
1 & 1 & 0 & 0 \\
0 & 0 & 1 & 1 \\
1 & 1 & 0 & 0 \\
0 & 0 & 1 & 1
\end{array}\right] \quad C=\left[\begin{array}{l}
a_{11} \\
a_{21} \\
a_{31} \\
a_{41}
\end{array}\right]
$$

The solution of the system for the image registration method is given by equation (4).

$$
C=B^{-1} A \text {. }
$$

After the determination of the polynomials, a third image is generated with the new obtained coordinates. This process is repeated using the resulting image, until the end of the scale. The final result consists in a complete line scale, as shown in Figure 5.

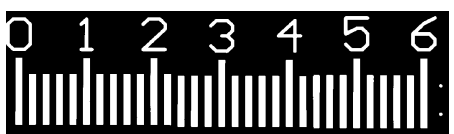

Fig. 5. Complete image of the scale after the registration.

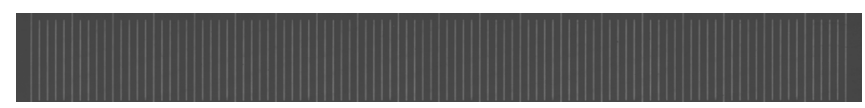

Fig. 6. Result of the registration process of a $1 \mathrm{~mm}$ scale.

\section{Results}

The purpose of using the image registration in line scale measurement is the determination of all the lengths in the scale quickly. This can be achieved from information obtained of the scale by the conventional method. With some previously measured values, it is possible to obtain the lengths in the scale. The methodology of measurement using image registration is not intended to replace the traditional methodology. Therefore, the scale calibration at certain points along its nominal length will continue to be done as they were before. These points measured by the conventional methodology will be used as reference values to obtain the remaining lengths of the scale by image registration.

The first scale used was the one previously shown in Figure 4. This scale has $6 \mathrm{~mm}$ nominal length and its smallest division is $0.2 \mathrm{~mm}$. To obtain the scale lengths results, the reference value used was at the $6 \mathrm{~mm}$ point, since it was presented on the scale calibration certificate. The measurements were obtained by counting the distances between all marks in pixel values. The conversion from pixels to millimeters is achieved by dividing the total length in millimeters from the measurement certificate $(6 \mathrm{~mm}$, in this case) by the same length, but found by counting the pixels from the first to the last mark. This mm/pixel ratio is then used as an estimate for the determination of the remaining lengths of the scale. The results are shown on Table 1.

The same procedure was used for a second scale, with different nominal length and different quantity of images registered to evaluate the efficiency of the method under different situations. This second scale has a nominal length of $1 \mathrm{~mm}$, and a distance between the marks of $0.01 \mathrm{~mm}$, Figure 6 shows the resulting image of this scale, registered from the merging of 10 images.

The reference for this scale is the calibration certificate. The size of the pixels on the image can be obtained by dividing the length informed on the scale certificate and the number of pixels counted on the registered image. Therefore, with this value, all the lengths for the scale are obtained as can be seen in Table 2 .

To evaluate the results, the values obtained through the registration method were compared with the lengths reported on the calibration certificate of the two scales. The parameter chosen to compare the results was the 
Table 1. Results obtained for the lengths of the $6 \mathrm{~mm}$ scale.

\begin{tabular}{|c|c|c|c|c|c|}
\hline $\begin{array}{c}\text { Nominal } \\
\text { value } \\
(\mathrm{mm}) \\
\end{array}$ & $\begin{array}{c}\text { Measured } \\
\text { value } \\
(\mathrm{mm})\end{array}$ & $\begin{array}{c}\text { Nominal } \\
\text { value } \\
(\mathrm{mm})\end{array}$ & $\begin{array}{c}\text { Measured } \\
\text { value } \\
(\mathrm{mm})\end{array}$ & $\begin{array}{c}\text { Nominal } \\
\text { value } \\
(\mathrm{mm})\end{array}$ & $\begin{array}{c}\text { Measured } \\
\text { value } \\
(\mathrm{mm})\end{array}$ \\
\hline 0.2 & 0.2018 & 2.2 & 2.1984 & 4.2 & 4.2016 \\
\hline 0.4 & 0.4003 & 2.4 & 2.3986 & 4.4 & 4.4017 \\
\hline 0.6 & 0.6005 & 2.6 & 2.5987 & 4.6 & 4.5986 \\
\hline 0.8 & 0.8006 & 2.8 & 2.8005 & 4.8 & 4.7971 \\
\hline 1.0 & 1.0008 & 3.0 & 2.9990 & 5.0 & 4.9973 \\
\hline 1.2 & 1.1976 & 3.2 & 3.1992 & 5.2 & 5.1958 \\
\hline 1.4 & 1.3978 & 3.4 & 3.3993 & 5.4 & 5.3943 \\
\hline 1.6 & 1.5979 & 3.6 & 3.5962 & 5.6 & 5.5912 \\
\hline 1.8 & 1.7981 & 3.8 & 3.7980 & 5.8 & 5.7897 \\
\hline 2.0 & 1.9982 & 4.0 & 3.9998 & 6.0 & 5.9915 \\
\hline
\end{tabular}

Table 2. Results obtained for the lengths of the $1 \mathrm{~mm}$ scale.

\begin{tabular}{cccccccc}
\hline $\begin{array}{c}\text { Nominal } \\
\text { value } \\
(\mathrm{mm})\end{array}$ & $\begin{array}{c}\text { Measured } \\
\text { value } \\
(\mathrm{mm})\end{array}$ & $\begin{array}{c}\text { Nominal } \\
\text { value } \\
(\mathrm{mm})\end{array}$ & $\begin{array}{c}\text { Measured } \\
\text { value } \\
(\mathrm{mm})\end{array}$ & $\begin{array}{c}\text { Nominal } \\
\text { value } \\
(\mathrm{mm})\end{array}$ & $\begin{array}{c}\text { Measured } \\
\text { value } \\
(\mathrm{mm})\end{array}$ & $\begin{array}{c}\text { Nominal } \\
\text { value } \\
(\mathrm{mm})\end{array}$ & $\begin{array}{c}\text { Measured } \\
\text { value } \\
(\mathrm{mm})\end{array}$ \\
\hline 0.01 & 0.0101 & 0.26 & 0.2607 & 0.51 & 0.5104 & 0.76 & 0.7603 \\
0.02 & 0.0200 & 0.27 & 0.2706 & 0.52 & 0.5205 & 0.77 & 0.7702 \\
0.03 & 0.0302 & 0.28 & 0.2806 & 0.53 & 0.5305 & 0.78 & 0.7803 \\
0.04 & 0.0402 & 0.29 & 0.2906 & 0.54 & 0.5406 & 0.79 & 0.7903 \\
0.05 & 0.0503 & 0.30 & 0.3006 & 0.55 & 0.5506 & 0.80 & 0.8001 \\
0.06 & 0.0602 & 0.31 & 0.3105 & 0.56 & 0.5606 & 0.81 & 0.8101 \\
0.07 & 0.0703 & 0.32 & 0.3206 & 0.57 & 0.5706 & 0.82 & 0.8201 \\
0.08 & 0.0804 & 0.33 & 0.3306 & 0.58 & 0.5806 & 0.83 & 0.8302 \\
0.09 & 0.0905 & 0.34 & 0.3407 & 0.59 & 0.5905 & 0.84 & 0.8402 \\
0.10 & 0.1003 & 0.35 & 0.3506 & 0.60 & 0.6004 & 0.85 & 0.8501 \\
0.11 & 0.1103 & 0.36 & 0.3607 & 0.61 & 0.6103 & 0.86 & 0.8602 \\
0.12 & 0.1204 & 0.37 & 0.3708 & 0.62 & 0.6203 & 0.87 & 0.8702 \\
0.13 & 0.1304 & 0.38 & 0.3808 & 0.63 & 0.6303 & 0.88 & 0.8802 \\
0.14 & 0.1404 & 0.39 & 0.3908 & 0.64 & 0.6403 & 0.89 & 0.8908 \\
0.15 & 0.1504 & 0.40 & 0.4007 & 0.65 & 0.6504 & 0.90 & 0.8999 \\
0.16 & 0.1604 & 0.41 & 0.4107 & 0.66 & 0.6603 & 0.91 & 0.9099 \\
0.17 & 0.1705 & 0.42 & 0.4206 & 0.67 & 0.6705 & 0.92 & 0.9198 \\
0.18 & 0.1806 & 0.43 & 0.4305 & 0.68 & 0.6805 & 0.93 & 0.9298 \\
0.19 & 0.1906 & 0.44 & 0.4406 & 0.69 & 0.6905 & 0.94 & 0.9399 \\
0.20 & 0.2005 & 0.45 & 0.4506 & 0.70 & 0.7003 & 0.95 & 0.9500 \\
0.21 & 0.2105 & 0.46 & 0.4608 & 0.71 & 0.7102 & 0.96 & 0.9600 \\
0.22 & 0.2205 & 0.47 & 0.4706 & 0.72 & 0.7207 & 0.97 & 0.9700 \\
0.23 & 0.2305 & 0.48 & 0.4808 & 0.73 & 0.7303 & 0.98 & 0.9801 \\
0.24 & 0.2405 & 0.49 & 0.4908 & 0.74 & 0.7402 & 0.99 & 0.9900 \\
0.25 & 0.2506 & 0.50 & 0.5005 & 0.75 & 0.7503 & 1.00 & 1.0000 \\
\hline
\end{tabular}

normalized error (EN) [11], calculated according to equation (5).

$$
E N=\left|\frac{V V-V M}{\sqrt{U_{V V}^{2}+U_{V M}^{2}}}\right|
$$

where:

$V V$ True value.

$V M$ Measured value.

$U_{V V}^{2}$ Expanded measurement uncertainty of the true value.

$U_{V M}^{2}$ Expanded measurement uncertainty of the measured value.
Table 3. Validation of the results for the image registration method of the $6 \mathrm{~mm}$ scale.

\begin{tabular}{cccc}
\hline $\begin{array}{c}\text { Nominal } \\
\text { value } \\
(\mathrm{mm})\end{array}$ & $\begin{array}{c}\text { Reference } \\
\text { value } \\
(\mathrm{mm})\end{array}$ & $\begin{array}{c}\text { Measured } \\
\text { value } \\
(\mathrm{mm})\end{array}$ & $E N$ \\
\hline 1.0 & 1.0008 & 0.9993 & 0.64 \\
2.0 & 1.9982 & 1.9990 & 0.35 \\
3.2 & 3.1992 & 3.1989 & 0.13 \\
4.0 & 3.9998 & 3.9982 & 0.72 \\
5.2 & 5.1958 & 5.1969 & 0.52 \\
6.0 & 5.9915 & 5.9915 & 0.00 \\
\hline
\end{tabular}

The expanded uncertainty $U_{V M}^{2}$ was estimated according to the guidelines of ISO GUM [ref], and for the $6 \mathrm{~mm}$ scale the value was $2 \mu \mathrm{m}$. Among the uncertainty sources, 
Table 4. Validation of the results for the image registration method of the $1 \mathrm{~mm}$ scale.

\begin{tabular}{cccc}
\hline $\begin{array}{c}\text { Nominal } \\
\text { value } \\
(\mathrm{mm})\end{array}$ & $\begin{array}{c}\text { Reference } \\
\text { value } \\
(\mathrm{mm})\end{array}$ & $\begin{array}{c}\text { Measured } \\
\text { value } \\
(\mathrm{mm})\end{array}$ & $E N$ \\
\hline 0.0 & 0.000000 & 0.000000 & 0.00 \\
0.1 & 0.099993 & 0.100339 & 0.34 \\
0.2 & 0.199981 & 0.200522 & 0.54 \\
0.3 & 0.299981 & 0.300600 & 0.62 \\
0.4 & 0.400027 & 0.400730 & 0.70 \\
0.5 & 0.500030 & 0.500494 & 0.46 \\
0.6 & 0.599936 & 0.600363 & 0.43 \\
0.7 & 0.699939 & 0.700336 & 0.40 \\
0.8 & 0.800000 & 0.800101 & 0.10 \\
0.9 & 0.899947 & 0.899917 & 0.03 \\
1.0 & 0.999995 & 0.999995 & 0.00 \\
\hline
\end{tabular}

the most influential ones were repeatability, temperature and pixel dimension. For the expanded measurement uncertainty of the true value the certificated result was used.

Table 4 shows the results for the $1 \mathrm{~mm}$ scale EN calculation. The expanded uncertainty for the image registration method in this scale was of $2 \mu \mathrm{m}$.

\section{Conclusions}

The results presented were considered satisfactory since in all cases the obtained normalized error was less than 1 . When compared to the scale calibration certificate, which makes valid the use of the image registration technique for the graduated scales measurement. The reproductibility of the method has been proven by measuring two different line scales and having satisfactory results for the normalized error.

From this implementation it becomes possible the determination of all the scales lengths quickly, which would be impracticable through the conventional methodology, due to the high amount of measured points.

For a future work, it is suggested the study of the measurement uncertainty, where all the error sources for the measurement would be evaluated within the image registration model, as well as contributions of the used standards, environmental conditions of measurement, among others.

\section{References}

1. J.S. Beers, W.B. Penzes, The NIST Lenght Scale Interferometer, J. Res. Natl. Inst. Stand. Technol. 104, 225-252 (1999)

2. A. Lassila, E. Ikonen, K. Risk, Interferometer for calibration of graduated line scales with a moving CCD camera as a line detector, Appl. Opt. 33, 3600-3603 (1994)

3. T. Eom, J. Han, A precision length measuring system for a variety of linear artefacts, Meas. Sci. Technol. 12, 678-701 (2001)

4. J.E. Deecker, A.G. Steele, H. Bosse, R.J. Douglas, Analysing redundancy in a line scale comparison using Monte Carlo methods, Meas. Sci. Technol. 19, 064005 (2008)

5. H. Bosse, W. Haessler-Grohne, J. Fluegge, R. Koening, Final report on CCL-S3 supplementary line scale comparison Nano 3, Metrologia 40, 04002 (2003)

6. B. Zitová, J. Flusser, F. Sroubek, Image Registration: A Survey and Recent Advances, ICIP 2005 Tutorial (Institute of Information Theory and Automation, Czech Republic, 2005)

7. A.A. Goshtasby, 2-D and 3-D Image Registration for Medical, Remote Sensing and Industrial Applications (Wiley-Interscience, 2005), p. 258

8. E. Ferandéz, R. Marti, GRASP for seam drawing in mosaicking of aerial photographic maps, J. Heuristics 5, 181197 (1999)

9. J. Hsieh, Fast Stitching algorithm for moving object detection and mosaic construction, Image Vision Computing 22, 291-306 (2004)

10. W.K. Pratt, Digital Image Processing (John Wiley, 2007), Vol. 4, p. 782

11. The Fitness for Purpose of Analytical Methods. A laboratory Guide to Method Validation and Related Topics, 1st edn. (EURACHEM, 1998) 